\section{Acute coronary syndromes: relationship between plasma lipoproteins and clinical outcome}

A new study by Olsson and colleagues has investigated the relationship between initial plasma lipoprotein levels and short-term prognosis in patients with acute coronary syndromes.

Part of the Myocardial Ischemia Reduction with Aggressive Cholesterol Lowering (MIRACL) study, this analysis enrolled 3,086 patients who were admitted to hospital with unstable angina or non-Q-wave acute myocardial infarction. All were randomly assigned to atorvastatin $80 \mathrm{mg} /$ day or placebo for 16 weeks. Plasma lipid and lipoprotein concentrations were determined at baseline, at 6 weeks and at 16 weeks. Additionally, apolipoproteins $B(a p o B)$ and $A-1$ (apoA-1) were measured at baseline and at 16 weeks.

As expected, atorvastatin treatment lowered LDL-cholesterol levels but had no significant effect on HDL cholesterol. However, LDL-cholesterol levels-either at baseline or on treatment-did not influence clinical outcome during the 16-week follow-up period. Conversely, baseline HDL cholesterol predicted the risk of recurrent cardiovascular events (hazard ratio 0.986 per $\mathrm{mg} / \mathrm{dl}$ increment in $\mathrm{HDL}$ cholesterol, $P<0.001$ ).

Olsson et al. suggest that these results point to a pleiotropic effect of statins, and they warn that measurements of serum lipoproteins should not guide the use of this therapy in patients with acute coronary syndromes.

Original article Olsson AG et al. (2005) High-density lipoprotein, but not low-density lipoprotein cholesterol levels influence short-term prognosis after acute coronary syndrome: results from the MIRACL trial. Eur Heart J 26: 890-896

\section{Noncontact mapping: guiding ablation of infarct-related ventricular tachycardia}

A recent study by Segal et al. has provided long-term follow-up data on the use of radiofrequency catheter ablation in patients with infarct-related ventricular tachycardia (VT).

The study was based on a percutaneous catheter-mounted noncontact mapping system (EnSite $3000^{\circledR}$, Endocardial Solutions Inc., St Paul, MN), which provided endocardial activation maps of the whole of the left ventricle. Guided by this system, the investigators performed radio-frequency ablation in 40 consecutive patients with infarct-related VT. The procedure was successfully completed in 33 cases $(82.5 \%)$. A total of $140 \mathrm{VTs}$ were mapped-36 (25.7\%) of which occurred spontaneously-and 81 were targeted for ablation using a mean of $6.5 \pm 6.2$ radio-frequency applications per patient. Ablation was successful in $67(82.7 \%)$ of the VTs targeted, including 27 (75\%) of the 36 clinical VTs.

Despite the high procedural success rate, only $17(42.5 \%)$ of the 40 patients remained free from VT and ventricular fibrillation during the follow-up period of $36.3 \pm 21.0$ months. The results indicate that this approach should not be viewed as a substitute for implantable cardioverter-defibrillators and antiarrhythmic drugs, but might be useful as an adjunctive therapy.

Original article Segal OR et al. (2005) Long-term results after ablation of infarct-related ventricular tachycardia. Heart Rhythm 2: 474-482

\section{Monocyte count predicts carotid plaque formation}

Since the differentiation of monocytes into macrophages is a key step in the development of atherosclerotic plaques, Johnsen et al. considered whether monocyte activity is associated with the risk of plaque formation in those without pre-existing disease. The results of their investigation have recently been published in Stroke.

This population-based analysis included 2,610 participants aged 25-82 years, all of whom were participants in the Troms $\varnothing$ Study in Norway. At baseline, ultrasound examination showed that all participants were free of plaques in the right carotid artery. Along with standard cardiovascular risk factors such as age and smoking, the investigators recorded the patients' baseline monocyte count, white cell count, fibrinogen, and intima-media thickness (IMT). A second ultrasound examination was performed 7 years later, and the incidence of plaques was correlated with the various risk factors in a multivariate analysis. 